\title{
Introduction to Hopf-Cyclic Cohomology
}

\author{
Masoud Khalkhali and Bahram Rangipour
}

\begin{abstract}
We review the recent progress in the study of cyclic cohomology in the presence of Hopf symmetry.
\end{abstract}

\section{Introduction}

In their study of the index theory of transversally elliptic operators 9, Connes and Moscovici develope a cyclic cohomology theory for Hopf algebras which can be regarded, post factum, as the right noncommutative analogue of group homology and Lie algebra homology. One of the main reasons for introducing this theory was to obtain a noncommutative characteristic map

$$
\chi_{\tau}: H C_{(\delta, \sigma)}^{*}(H) \longrightarrow H C^{*}(A),
$$

for an action of a Hopf algebra $H$ on an algebra $A$ endowed with an "invariant trace" $\tau: A \rightarrow \mathbb{C}$. Here, the pair $(\delta, \sigma)$ consists of a grouplike element $\sigma \in H$ and a characater $\delta: H \rightarrow \mathbb{C}$ satisfying certain compatibility conditions explained in Section 2.3 below.

In 21] we found a new approach to this subject and extended it, among other things, to a cyclic cohomology theory for triples $(C, H, M)$, where $C$ is a coalgebra endowed with an action of a Hopf algebra $H$ and $M$ is an $H$ module and an $H$-comodule satisfying some extra compatibility conditions. It was observed that the theory of Connes and Moscovici corresponds to $C=H$ equipped with the regular action of $H$ and $M$ a one dimensional $H$-module with an extra structure.

One of the main ideas of [21] was to view the Hopf-cyclic cohomology as the cohomology of the invariant part of certain natural complexes attached to $(C, H, M)$. This is remarkably similar to interpreting the cohomology of the Lie algebra of a Lie group as the invariant part of the de Rham cohomology 
of the Lie group. The second main idea was to introduce coefficients into the theory. This also explained the important role played by the so called modular pair $(\delta, \sigma)$ in $[9]$.

Since the module $M$ is a noncommutative analogue of coefficients for Lie algebra and group homology theories, it is of utmost importance to understand the most general type of coefficients allowable. In fact the periodicity condition $\tau_{n}^{n+1}=i d$ for the cyclic operator and the fact that all simplicial and cyclic operators have to descend to the invrainat complexes, puts very stringent conditions on the type of the $H$-module $M$. This problem that remained unsettled in our paper [21] is completely solved in HajacKhalkhali-Rangipour-Sommerhäuser papers 14, 15] by introducing the class of stable anti-Yetter-Drinfeld modules over a Hopf algebra. The category of anti-Yetter-Drinfeld modules over a Hopf algebra $H$ is a twisting, or 'mirror image' of the category of Yetter-Drinfeld $H$-modules. Technically it is obtained from the latter by replacing the antipode $S$ by $S^{-1}$ although this connection is hardly illuminating.

In an effort to make this paper more accessible, we cover basic background material, with many examples, on Hopf algebras and the emerging role of Hopf symmetry in noncommutative geometry and its applications [9, 10, 11, 12. This is justified since certain doses of the "yoga of Hopf algebras", in the noncommutative and non-cocommutative case, is necessary to understand these works. Following these works, we emphasize the universal role played by the Connes-Moscovici Hopf algebra $\mathcal{H}_{1}$ and its cyclic cohomology in applications of noncommutative geometry to transverse geometry and number theory. See also Marcolli's article 24] as well as Connes-Marcolli articles [6, 7] and references therein for recent interactions between number theory and noncommutative geometry.

It is a great pleasure to thank Alain Connes and Henri Moscovici for their support and many illuminating discussions, and Matilde Marcolli for organizing the MPI, Bonn, conferences on noncommutative geometry and number theory and for her support. We are also much obliged to our collaborators Piotr M. Hajac and Yorck Sommerhäuser.

\section{Preliminaries}

In this section we recall some aspects of Hopf algebra theory that are most relevant to the current status of Hopf-cyclic cohomology theory. 


\subsection{Coalgebras, bialgebras, and Hopf algebras}

We assume our Hopf algebras, coalgebras, and algebras are over a fixed field $k$ of characteristic zero. Most of our definitions and constructions however continue to work over an arbitrary commutative ground ring $k$. Unadorned $\otimes$ and Hom will always be over $\mathrm{k}$ and $I$ will always denote an identity map whose domain will be clear from the context. By a coalgebra over $k$ we mean a $k$-linear space $C$ endowed with $k$-linear maps

$$
\Delta: C \longrightarrow C \otimes C, \quad \varepsilon: C \longrightarrow k
$$

called comultiplication and counit respectively, such that $\Delta$ is coassociative and $\varepsilon$ is the counit of $\Delta$. That is,

$$
\begin{gathered}
(\Delta \otimes I) \circ \Delta=(I \otimes \Delta) \circ \Delta: C \longrightarrow C \otimes C \otimes C, \\
(\varepsilon \otimes I) \circ \Delta=(I \otimes \varepsilon) \circ \Delta=I .
\end{gathered}
$$

$C$ is called cocommutative if $\tau \Delta=\Delta$, where $\tau: C \otimes C \rightarrow C \otimes C$ is the fillip $x \otimes y \mapsto y \otimes x$.

We use Sweedler-Heynemann's notation for comultiplication, with summation suppressed, and write

$$
\Delta(c)=c^{(1)} \otimes c^{(2)}
$$

With this notation the axioms for a coalgebra read as

$$
\begin{gathered}
c^{(1)} \otimes c^{(2)(1)} \otimes c^{(2)(2)}=c^{(1)(1)} \otimes c^{(1)(2)} \otimes c^{(2)}, \\
\varepsilon\left(c^{(1)}\right)\left(c^{(2)}\right)=c=\left(c^{(1)}\right) \varepsilon\left(c^{(2)}\right),
\end{gathered}
$$

for all $c \in C$. We put

$$
c^{(1)} \otimes c^{(2)} \otimes c^{(3)}:=(\Delta \otimes I) \Delta(c) .
$$

Similarly, for iterated comultiplication maps

$$
\Delta^{n}:=(\Delta \otimes I) \circ \Delta^{n-1}: C \longrightarrow C^{\otimes(n+1)}, \quad \Delta^{1}=\Delta,
$$

we write

$$
\Delta^{n}(c)=c^{(1)} \otimes \cdots \otimes c^{(n+1)},
$$


where summation is understood. Many notions for algebras have their dual analogues for coalgebras. Thus, one can easily define such notions like, subcoalgebra, (left, right, two sided) coideal, quotient coalgebra, and morphism of coalgebras [26, 29].

A left $C$-comodule is a linear space $M$ together with a linear map $\rho$ : $M \rightarrow C \otimes M$ such that $(\rho \otimes 1) \rho=\Delta \rho$ and $(\varepsilon \otimes 1) \rho=\rho$. We write

$$
\rho(m)=m^{(-1)} \otimes m^{(0)}
$$

where summation is understood, to denote the coaction $\rho$. Similarly if $M$ is a right $C$-comodule, we write

$$
\rho(m)=m^{(0)} \otimes m^{(1)}
$$

to denote its coaction $\rho: M \rightarrow M \otimes C$. Note that module elements are always assigned zero index. Let $M$ and $N$ be left $C$-comodules. A $C$-colinear map is a linear map $f: M \rightarrow N$ such that $\rho_{N} f=(1 \otimes f) \rho_{M}$. The category of left $C$-comodules is an abelian category; note that, unlike the situation for algebras, for this to be true, it is important that $C$ be a flat $k$-module which will be the case if $k$ is a field.

Let $C$ be a coalgebra, $A$ be a unital algebra, and $f, g: C \rightarrow A$ be $k$-linear maps. The convolution product of $f$ and $g$, denoted by $f * g$, is defined as the composition

$$
C \stackrel{\Delta}{\longrightarrow} C \otimes C \stackrel{f \otimes g}{\longrightarrow} A \otimes A,
$$

or equivalently by

$$
(f * g)(c)=f\left(c^{(1)}\right) g\left(c^{(2)}\right) .
$$

It is easily checked that under the convolution product $\operatorname{Hom}(C, A)$ is an associative unital algebra. Its unit is the map $e: C \rightarrow A, e(c)=\varepsilon(c) 1_{A}$. In particular the linear dual of a coalgebra $C, C^{*}=\operatorname{Hom}(C, k)$, is an algebra.

A bialgebra is a unital algebra endowed with a compatible coalgebra structure. This means that the coalgebra structure maps $\Delta: B \longrightarrow B \otimes B, \quad \varepsilon$ : $B \longrightarrow k$, are morphisms of unital algebras. This is equivalent to multiplication and unit maps of $A$ being morphisms of coalgebras.

A Hopf algebra is a bialgebra endowed with an antipode. By definition, an antipode for a bialgebra $H$ is a linear map $S: H \rightarrow H$ such that

$$
S * I=I * S=\eta \varepsilon,
$$


where $\eta: k \rightarrow H$ is the unit map of $H$. Equivalently,

$$
S\left(h^{(1)}\right) h^{(2)}=h^{(1)} S\left(h^{(2)}\right)=\varepsilon(h) 1,
$$

for all $h \in H$. Thus $S$ is the inverse of the identity map $I: H \rightarrow H$ in the convolution algebra $\operatorname{Hom}(H, H)$. This shows that the antipode is unique, if it exists at all. The following properties of the antipode are well known:

1. If $H$ is commutative or cocommutative then $S^{2}=I$. The converse need not be true.

2. $\mathrm{S}$ is an anti-algebra map and an anti-coalgebra map. The latter means

$$
S\left(h^{(2)}\right) \otimes S\left(h^{(1)}\right)=S(h)^{(1)} \otimes S(h)^{(2)},
$$

for all $h \in H$.

We give a few examples of Hopf algebras:

1. Commutative or cocommutative Hopf algebras are closely related to groups and Lie algebras. We give a few examples to indicate this connection

1.a. Let $G$ be a discrete group (need not be finite) and $H=k G$ the group algebra of $G$ over $k$. Let

$$
\Delta(g)=g \otimes g, \quad S(g)=g^{-1}, \quad \text { and } \varepsilon(g)=1,
$$

for all $g \in G$ and linearly extend them to $H$. Then it is easy to check that $(H, \Delta, \varepsilon, S)$ is a cocommutative Hopf algebra. It is of course commutative if and only if $G$ is commutative.

1.b. Let $\mathfrak{g}$ be a $k$-Lie algebra and $H=U(\mathfrak{g})$ be the universal enveloping algebra of $\mathfrak{g}$. Using the universal property of $U(\mathfrak{g})$ one checks that there are uniquely defined algebra homomorphisms $\Delta: U(\mathfrak{g}) \rightarrow U(\mathfrak{g}) \otimes U(\mathfrak{g})$, $\varepsilon: U(\mathfrak{g}) \rightarrow k$ and an anti-algebra map $S: U(\mathfrak{g}) \rightarrow U(\mathfrak{g})$, determined by

$$
\Delta(X)=X \otimes 1+1 \otimes X, \quad \varepsilon(X)=0, \quad \text { and } S(X)=-X,
$$

for all $X \in \mathfrak{g}$. One then checks easily that $(U(\mathfrak{g}), \Delta, \varepsilon, S)$ is a cocommutative Hopf algebra. It is commutative if and only if $\mathfrak{g}$ is an abelian Lie algebra, in which case $U(\mathfrak{g})=S(\mathfrak{g})$ is the symmetric algebra of $\mathfrak{g}$. 
1.c. Let $G$ be a compact topological group. A continuous function $f$ : $G \rightarrow \mathbb{C}$ is called representable if the set of left translates of $f$ by all elements of $G$ forms a finite dimensional subspace of the space $C(G)$ of all continuous complex valued functions on $G$. It is then easily checked that the set of all representable functions, $H=\operatorname{Rep}(G)$, is a subalgebra of the algebra of continuous functions on $G$. Let $m: G \times G \rightarrow G$ denote the multiplication of $G$ and $m^{*}: C(G \times G) \rightarrow C(G), m^{*} f(x, y)=f(x y)$, denote its dual map. It is easy to see that if $f$ is representable, then $m^{*} f \in \operatorname{Rep}(G) \otimes \operatorname{Rep}(G) \subset$ $C(G \times G)$. Let $e$ denote the identity of $G$. One can easily check that the relations

$$
\Delta f=m^{*} f, \quad \varepsilon f=f(e), \quad \text { and }(S f)(g)=f\left(g^{-1}\right),
$$

define a Hopf algebra structure on $\operatorname{Rep}(G)$. Alternatively, one can describe $\operatorname{Rep}(G)$ as the linear span of matrix coefficients of all finite dimensional complex representations of $G$. By Peter-Weyl's Theorem, $\operatorname{Rep}(G)$ is a dense subalgebra of $C(G)$. This algebra is finitely generated (as an algebra) if and only if $G$ is a Lie group.

1.d. The coordinate ring of an affine algebraic group $H=k[G]$ is a commutative Hopf algebra. The maps $\Delta, \varepsilon$, and $S$ are the duals of the multiplication, unit, and inversion maps of $G$, respectively. More generally, an affine group scheme, over a commutative ring $k$, is a commutative Hopf algebra over $k$. Given such a Hopf algebra $H$, it is easy to see that for any commutative $k$-algebra $A$, the set $H_{\text {om }}$ Alg $(H, A)$ is a group under convolution product and $A \mapsto \operatorname{Hom}_{A l g}(H, A)$ is a functor from the category $\mathrm{ComAlg}_{k}$ of commutative $k$-algebras to the category of groups. Conversely, any representable functor Com Alg $\mathrm{Cl}_{k} \rightarrow$ Groups is represented by a, unique up to isomorphism, commutative $k$-Hopf algebra. Thus the category of affine group schemes is equivalent to the category of representable functors ComAlg $\mathrm{Co}_{k} \rightarrow$ Groups.

2. Compact quantum groups and quantized enveloping algebras are examples of noncommutative and noncommutative Hopf algebras [26]. We won't recall these examples here. A very important example for noncommutative geometry and its applications to transverse geometry and number theory is the Connes-Moscovici Hopf algebra $\mathcal{H}_{1}$ [9, 11, 12] which we recall now. Let $\mathfrak{g}_{a f f}$ be the Lie algebra of the group of affine transformations of the line with linear basis $X$ and $Y$ and the relation $[Y, X]=X$. Let $\mathfrak{g}$ be an abelian Lie 
algebra with basis $\left\{\delta_{n} ; n=1,2, \cdots\right\}$. It is easily seen that $\mathfrak{g}_{\text {aff }}$ acts on $\mathfrak{g}$ via

$$
\left[Y, \delta_{n}\right]=n \delta_{n}, \quad\left[X, \delta_{n}\right]=\delta_{n+1},
$$

for all $n$. Let $\mathfrak{g}_{C M}:=\mathfrak{g}_{a f f} \rtimes \mathfrak{g}$ be the corresponding semidirect product Lie algebra. As an algebra, $\mathcal{H}_{1}$ coincides with the universal enveloping algebra of the Lie algebra $\mathfrak{g}_{C M}$. Thus $\mathcal{H}_{1}$ is the universal algebra generated by $\left\{X, Y, \delta_{n} ; n=1,2, \cdots\right\}$ subject to relations

$$
[Y, X]=X, \quad\left[Y, \delta_{n}\right]=n \delta_{n}, \quad\left[X, \delta_{n}\right]=\delta_{n+1}, \quad\left[\delta_{k}, \delta_{l}\right]=0,
$$

for $n, k, l=1,2, \cdots$. We let the counit of $\mathcal{H}_{1}$ coincide with the counit of $U\left(\mathfrak{g}_{C M}\right)$. Its coproduct and antipode, however, will be certain deformations of the coproduct and antipode of $U\left(\mathfrak{g}_{C M}\right)$ as follows. Using the universal property of $U\left(\mathfrak{g}_{C M}\right)$, one checks that the relations

$$
\begin{gathered}
\Delta Y=Y \otimes 1+1 \otimes Y, \quad \Delta \delta_{1}=\delta_{1} \otimes 1+1 \otimes \delta_{1}, \\
\Delta X=X \otimes 1+1 \otimes X+\delta_{1} \otimes Y,
\end{gathered}
$$

determine a unique algebra map $\Delta: \mathcal{H}_{1} \rightarrow \mathcal{H}_{1} \otimes \mathcal{H}_{1}$. Note that $\Delta$ is not cocommutative and it differs from the corrodent of the enveloping algebra $U\left(\mathfrak{g}_{C M}\right)$. Similarly, one checks that there is a unique antialgebra map $S$ : $\mathcal{H}_{1} \rightarrow \mathcal{H}_{1}$ determined by the relations

$$
S(Y)=-Y, \quad S(X)=-X+\delta_{1} Y, \quad S\left(\delta_{1}\right)=-\delta_{1} .
$$

Again we note that this antipode also differs from the antipode of $U\left(\mathfrak{g}_{C M}\right)$, and in particular $S^{2} \neq I$. In fact $S^{n} \neq I$ for all $n$. In the next section we will show, following Connes-Moscovici [9], that $\mathcal{H}_{1}$ is a bicrossed product of Hopf algebras $U\left(\mathfrak{g}_{a f f}\right)$ and $U(\mathfrak{g})^{*}$, where $\mathfrak{g}$ is a pro-nilpotent Lie algebra to be described precisely in the next section.

Let $H$ be a Hopf algebra. A grouplike element of $H$ is a non-zero element $g \in H$ such that $\Delta g=g \otimes g$. We have, from the axioms for the antipode, $g S(g)=S(g) g=1_{H}$ which shows that $g$ is invertible. It is easily seen that grouplike elements of $H$ form a subgroup of the multiplicative group of $H$. For example, for $H=k G$ the set of grouplike elements coincide with the group $G$ itself. A primitive element is an element $x \in H$ such that $\Delta x=1 \otimes x+x \otimes 1$. It is easily seen that the bracket $[x, y]:=x y-y x$ of two primitive elements is again a primitive element. It follows that primitive 
elements form a Lie algebra. Using the Poincare-Birkhoff-Witt theorem, one shows that the set of primitive elements of $H=U(\mathfrak{g})$ coincide with the Lie algebra $\mathfrak{g}$.

A character of a Hopf algebra is a unital algebra map $\delta: H \rightarrow k$. For example the counit $\varepsilon: H \rightarrow k$ is a character. For a less trivial example, let $G$ be a non-unimodular real Lie group and $H=U(\mathfrak{g})$ the universal enveloping algebra of the Lie algebra of $G$. The modular function of $G$, measuring the difference between the right and left Haar measure on $G$, is a smooth group homomorphism $\Delta: G \rightarrow \mathbb{R}^{+}$. Its derivative at identity defines a Lie algebra map $\delta: \mathfrak{g} \rightarrow \mathbb{R}$. We denote its natural extension by $\delta: U(\mathfrak{g}) \rightarrow \mathbb{R}$. It is obviously a character of $U(\mathfrak{g})$. For a concrete example, let $G=\operatorname{Aff}(\mathbb{R})$ be the group of affine transformations of the real line. It is a non-unimodular group with modular homomorphism given by

$$
\Delta\left(\begin{array}{ll}
a & b \\
0 & 1
\end{array}\right)=|a|
$$

The corresponding infinitesimal character on $\mathfrak{g}_{a f f}=\operatorname{Lie}(G)$ is given by

$$
\delta(Y)=1, \quad \delta(X)=0
$$

where $Y=\left(\begin{array}{ll}1 & 0 \\ 0 & 0\end{array}\right)$ and $X=\left(\begin{array}{ll}0 & 1 \\ 0 & 0\end{array}\right)$ are a basis for $\mathfrak{g}_{a f f}$. We will see that this character plays an important role in constructing a twisted antipode for the Connes-Moscovici Hopf algebra $\mathcal{H}_{1}$.

If $H$ is a Hopf algebra, by a left $H$-module (resp. left $H$-comodule), we mean a left module (resp. left comodule) over the underlying algebra (resp. the underlying coalgebra) of $H$.

Recall that a monoidal, or tensor, category $(\mathcal{C}, \otimes, U, a, l, r)$ consists of a category $\mathcal{C}$, a functor $\otimes: \mathcal{C} \times \mathcal{C} \rightarrow \mathcal{C}$, an object $U \in \mathcal{C}$ (called the unit object), and natural isomorphisms

$$
\begin{gathered}
a=a_{A, B, C}: A \otimes(B \otimes C) \rightarrow(A \otimes B) \otimes C, \\
l=l_{A}: U \otimes A \rightarrow A, \quad r=r_{A}: A \otimes U \rightarrow A,
\end{gathered}
$$

(called the associativity and unit constraints, respectively) such that the so called pentagon and triangle diagrams commute: 


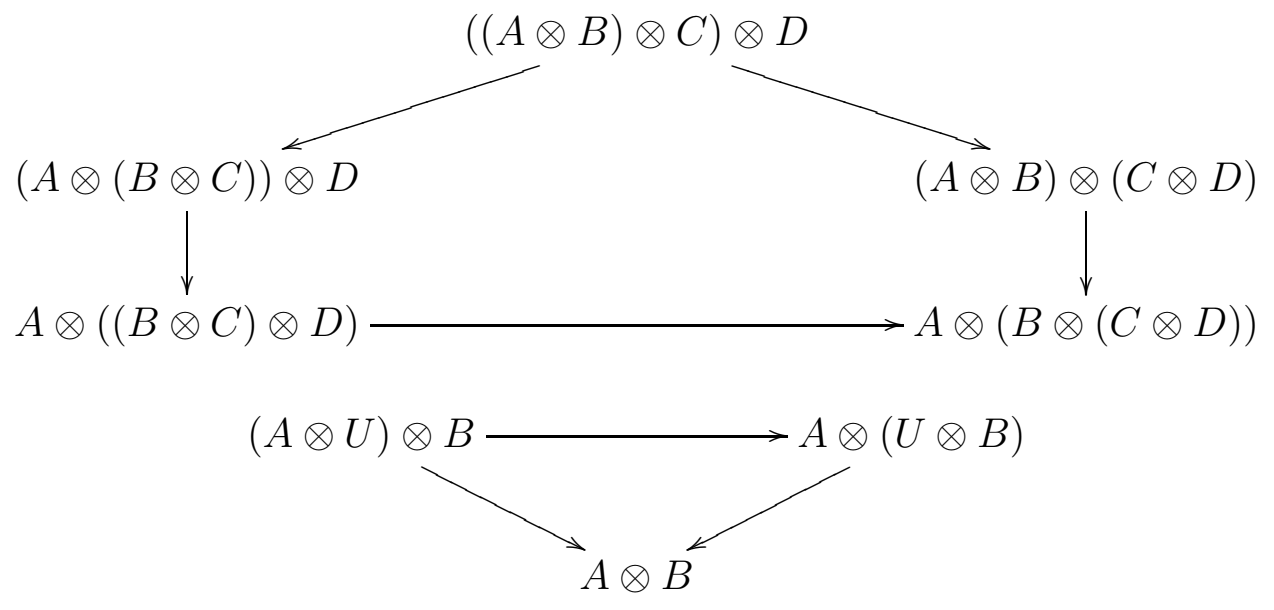

The coherence theorem of MacLane 25] guarantees that all diagrams composed from $a, l, r$ by tensoring, substituting and composing, commute.

A braided monoidal category, is a monoidal category $\mathcal{C}$ endowed with a natural family of isomorphisms

$$
c_{A, B}: A \otimes B \rightarrow B \otimes A,
$$

called braiding such that the following diagram and the one obtained from it by replacing $c$ by $c^{-1}$ commute (Hexagon axioms):

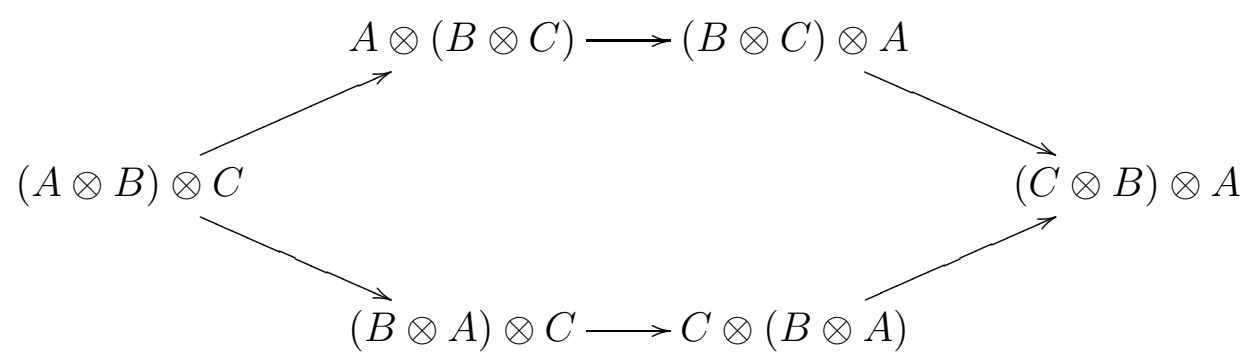

A braiding is called a symmetry if we have

$$
c_{A, B} \circ c_{B, A}=I
$$

for all $A, B$. A symmetric monoidal category, is a monoidal category endowed with a symmetry. 
Let $H$ be a bialgebra. Then thanks to the existence of a comultiplication on $H$, the category $H-M o d$ of left $H$-modules is a monoidal category: if $M$ and $N$ are left $H$-modules, their tensor product over $k, M \otimes N$, is again an $H$-module via

$$
h(m \otimes n)=h^{(1)} m \otimes h^{(2)} n .
$$

One can easily check that with associativity constraints defined as the natural isomorphism of $H$-modules $M \otimes(N \otimes P) \rightarrow(M \otimes N) \otimes P, m \otimes(n \otimes p) \mapsto$ $(m \otimes n) \otimes p$, and with unit object $U=k$ with trivial $H$-action via the counit $\varepsilon$

$$
h(1)=\varepsilon(h) 1,
$$

$H-\operatorname{Mod}$ is a monoidal category. If $H$ is cocommutative, then one checks that the map

$$
c_{M, N}: M \otimes N \rightarrow N \otimes M, \quad c_{M, N}(m \otimes n)=n \otimes m,
$$

is a morphism of $H$-modules and is a symmetry operator on $H-M o d$, turning it into a symmetric monoidal category.

The category $H-M o d$ is not braided in general. For that to happen, one must either restrict the class of modules to what is called Yetter-Drinfeld modules, or restrict the class of Hopf algebras to quasisimilar Hopf algebras to obtain a braiding on $H-\operatorname{Mod}$ [26]. We will discuss the first scenario in the next section and will see that, quite unexpectedly, this question is closely related to Hopf-cyclic cohomology.

Similarly, the category $H-C o m o d$ of left $H$-comodules is a monoidal category: if $M$ and $N$ are left $H$-comodules, their tensor product $M \otimes N$ is again an $H$-comodule via

$$
\rho(m \otimes n)=m^{(-1)} n^{(-1)} \otimes m^{(0)} \otimes n^{(0)} .
$$

Its unit object is $U=k$ endowed with the $H$-coaction $r \in k \mapsto 1_{H} \otimes r$. If $H$ is commutative then $H-C o m o d$ is a symmetric monoidal category. More generally, when $H$ is co-quasitriangular, $H-C o m o d$ can be endowed with a braiding [26].

\subsection{Symmetry in noncommutative geometry}

The idea of symmetry in noncommutative geometry is encoded by the action or coaction of a Hopf algebra on an algebra or on a coalgebra. Thus there are 
four possibilities in general that will be referred to as (Hopf-) module algebra, module coalgebra, comodule algebra, and comodule coalgebra (for each type, of course, we still have a choice of left or right action or coaction). We call them symmetries of type $A, B, C$ and $D$, respectively. We will see in the next sections that associated to each type of symmetry there is a corresponding cyclic cohomology theory with coefficients. These theories in a certain sense are generalizations of equivariant de Rham cohomology with coefficients in an equivariant local system.

Let $H$ be a Hopf algebra. An algebra $A$ is called a left $H$-module algebra, if it is a left $H$-module and the multiplication map $A \otimes A \rightarrow A$ and the unit map $k \rightarrow A$ are morphisms of $H$-modules. This means

$$
h(a b)=h^{(1)}(a) h^{(2)}(b), \quad \text { and } \quad h(1)=\varepsilon(h) 1,
$$

for all $h \in H$ and $a, b \in A$ (summation is understood). Using the relations $\Delta g=g \otimes g$ and $\Delta x=1 \otimes x+x \otimes 1$, it is easily seen that in an $H$-module algebra, grouplike elements act as unit preserving automorphisms while primitive elements act as derivations. In particular, for $H=k G$ the group algebra of a discrete group, an $H$-module algebra structure on $A$ is simply an action of $G$ by unit preserving automorphisms on $A$. Similarly, we have a 1-1 correspondence between $U(\mathfrak{g})$-module algebra structures on $A$ and Lie actions of the Lie algebra $\mathfrak{g}$ by derivations on $A$.

An algebra $B$ is called a left $H$-comodule algebra, if $B$ is a left $H$-comodule and the multiplication and unit maps of $B$ are $H$-comodule maps. That is

$$
(a b)^{(-1)} \otimes(a b)^{(0)}=a^{(-1)} b^{(-1)} \otimes a^{(0)} b^{(0)}, \quad\left(1_{B}\right)^{(-1)} \otimes\left(1_{B}\right)^{(0)}=1_{H} \otimes 1_{B},
$$

for all $a, b$ in $B$.

A left $H$-module coalgebra is a coalgebra $C$ which is a left $H$-module such that the comultiplication map $\Delta: C \rightarrow C \otimes C$ and the counit map $\varepsilon: C \rightarrow k$ are $H$-module maps. That is

$$
(h c)^{(1)} \otimes(h c)^{(2)}=h^{(1)} c^{(1)} \otimes h^{(2)} c^{(2)}, \quad \varepsilon(h c)=\varepsilon(h) \varepsilon(c),
$$

for all $h \in H$ and $c \in C$.

Finally, a coalgebra $D$ is called a left $H$-comodule coalgebra when the comultiplication and counit maps of $D$ are morphisms of $H$-comodules. That is

$$
c^{(1)(-1)} c^{(2)(-1)} \otimes c^{(1)(0)} \otimes c^{(2)(0)}=c^{(-1)} \otimes c^{(0)(1)} \otimes c^{(0)(2)},
$$




$$
\varepsilon(c) 1_{H}=c^{(-1)} \varepsilon\left(c^{(0)}\right),
$$

for all $c \in C$.

We call the above four types of symmetries, symmetries of type $A, B$, $C$, and $D$, respectively. All four types of symmetries are present within an arbitrary Hopf algebra. For example, the coproduct $\Delta: H \rightarrow H \otimes H$ gives $H$ the structure of a left (and right) $H$-comodule algebra while the product $H \otimes H \rightarrow H$ turns $H$ into a left (and right) $H$-module coalgebra. These are noncommutative analogues of translation action of a group on itself. The conjugation action $H \otimes H \rightarrow H, g \otimes h \mapsto g^{(1)} h S\left(g^{(2)}\right)$ gives $H$ the structure of a left $H$-module algebra. The co-conjugation action $H \rightarrow H \otimes H$, $h \mapsto h^{(1)} S\left(h^{(3)}\right) \otimes h^{(2)}$ turns $H$ into an $H$-comodule coalgebra.

For a different example, we turn to the Connes-Moscovici Hopf algebra $\mathcal{H}_{1}$. An important feature of $\mathcal{H}_{1}$, and in fact its raison d'être, is that it acts as quantum symmetries of various objects of interest in noncommutative geometry, like the 'space' of leaves of codimension one foliations or the 'space' of modular forms modulo the action of Hecke correspondences. Let $M$ be a one dimensional manifold and $A=C_{0}^{\infty}\left(F^{+} M\right)$ denote the algebra of smooth functions with compact support on the bundle of positively oriented frames on $M$. Given a discrete group $\Gamma \subset \operatorname{Diff}^{+}(M)$ of orientation preserving diffeomorphisms of $M$, one has a natural prolongation of the action of $\Gamma$ to $F^{+}(M)$ by

$$
\varphi\left(y, y_{1}\right)=\left(\varphi(y), \varphi^{\prime}(y)\left(y_{1}\right)\right) .
$$

Let $A_{\Gamma}=C_{0}^{\infty}\left(F^{+} M\right) \rtimes \Gamma$ denote the corresponding crossed product algebra. Thus the elements of $A_{\Gamma}$ consist of finite linear combinations (over $\mathbb{C}$ ) of terms $f U_{\varphi}^{*}$ with $f \in C_{0}^{\infty}\left(F^{+} M\right)$ and $\varphi \in \Gamma$. Its product is defined by

$$
f U_{\varphi}^{*} \cdot g U_{\psi}^{*}=(f \cdot \varphi(g)) U_{\psi \varphi}^{*} .
$$

There is an action of $\mathcal{H}_{1}$ on $A_{\Gamma}$ given by [9, 12]:

$$
\begin{gathered}
Y\left(f U_{\varphi}^{*}\right)=y_{1} \frac{\partial f}{\partial y_{1}} U_{\varphi}^{*}, \quad X\left(f U_{\varphi}^{*}\right)=y_{1} \frac{\partial f}{\partial y} U_{\varphi}^{*}, \\
\delta_{n}\left(f U_{\varphi}^{*}\right)=y_{1}^{n} \frac{d^{n}}{d y^{n}}\left(\log \frac{d \varphi}{d y}\right) f U_{\varphi}^{*} .
\end{gathered}
$$

Once these formulas are given, it can be checked, by a long computation, that $A_{\Gamma}$ is indeed an $\mathcal{H}_{1}$-module algebra. In the original application, $M$ is a 
transversal for a codimension one foliation and thus $\mathcal{H}_{1}$ acts via transverse differential operators 9 .

We recall, very briefly, the action of the Hopf algebra $\mathcal{H}_{1}$ on the so called modular Hecke algebras, discovered by Connes and Moscovici in [12, 13] where detailed discussions and a very intriguing dictionary comparing transverse geometry notions with modular forms notions can be found. For each $N \geq 1$, let

$$
\Gamma(N)=\left\{\left(\begin{array}{ll}
a & b \\
c & d
\end{array}\right) \in S L(2, \mathbb{Z}) ; \quad\left(\begin{array}{ll}
a & b \\
c & d
\end{array}\right)=\left(\begin{array}{ll}
1 & 0 \\
0 & 1
\end{array}\right) \bmod N\right\}
$$

denote the level $N$ congruence subgroup of $\Gamma(1)=S L(2, \mathbb{Z})$. Let $\mathcal{M}_{k}(\Gamma(N))$ denote the space of modular forms of level $N$ and weight $k$ and

$$
\mathcal{M}(\Gamma(N)):=\oplus_{k} \mathcal{M}_{k}(\Gamma(N))
$$

be the graded algebra of modular forms of level $N$. Finally let

$$
\mathcal{M}:=\underset{\vec{N}}{\lim } \mathcal{M}(\Gamma(N))
$$

denote the algebra of modular forms of all levels, where the inductive system is defined by divisibility. The group

$$
G^{+}(\mathbb{Q}):=G L^{+}(2, \mathbb{Q}),
$$

acts on $\mathcal{M}$ by its usual action on functions on the upper half plane (with corresponding weight):

$$
\begin{gathered}
\left.(f, \alpha) \mapsto f\right|_{k} \alpha(z)=\operatorname{det}(\alpha)^{k / 2}(c z+d)^{-k} f(\alpha \cdot z), \\
\alpha=\left(\begin{array}{ll}
a & b \\
c & d
\end{array}\right), \quad \alpha . z=\frac{a z+b}{c z+d} .
\end{gathered}
$$

The elements of the corresponding crossed-product algebra

$$
\mathcal{A}=\mathcal{A}_{G^{+}(\mathbb{Q})}:=\mathcal{M} \rtimes G^{+}(\mathbb{Q}),
$$

are finite sums

$$
\sum f U_{\gamma}^{*}, \quad f \in \mathcal{M}, \quad \gamma \in G^{+}(\mathbb{Q}),
$$

with a product defined by

$$
f U_{\alpha}^{*} \cdot g U_{\beta}^{*}=(f \cdot g \mid \alpha) U_{\beta \alpha}^{*} .
$$


$\mathcal{A}$ can be thought of as the algebra of 'noncommutative coordinates' on the 'noncommutative quotient space' of modular forms modulo Hecke correspondences [12].

Consider the operator $X$ of degree two on the space of modular forms defined by

$$
X:=\frac{1}{2 \pi i} \frac{d}{d z}-\frac{1}{12 \pi i} \frac{d}{d z}(\log \Delta) \cdot Y
$$

where

$$
\Delta(z)=(2 \pi)^{12} \eta^{24}(z)=(2 \pi)^{12} q \prod_{n=1}^{\infty}\left(1-q^{n}\right)^{24}, \quad q=e^{2 \pi i z}
$$

and $Y$ denotes the grading operator

$$
Y(f)=\frac{k}{2} \cdot f, \quad \text { for all } f \in \mathcal{M}_{k}
$$

The following proposition is proved in $\left[12\right.$. It shows that $\mathcal{A}_{G^{+}(\mathbb{Q})}$ is an $\mathcal{H}_{1^{-}}$ module algebra:

Proposition 2.1. There is a unique action of $\mathcal{H}_{1}$ on $\mathcal{A}_{G^{+}(\mathbb{Q})}$ determined by

$$
\begin{gathered}
X\left(f U_{\gamma}^{*}\right)=X(f) U_{\gamma}^{*}, \quad Y\left(f U_{\gamma}^{*}\right)=Y(f) U_{\gamma}^{*}, \\
\delta_{1}\left(f U_{\gamma}^{*}\right)=\mu_{\gamma} \cdot f\left(U_{\gamma}^{*}\right),
\end{gathered}
$$

where

$$
\mu_{\gamma}(z)=\frac{1}{2 \pi i} \frac{d}{d z} \log \frac{\Delta \mid \gamma}{\Delta}
$$

More generally, for any congruence subgroup $\Gamma$ an algebra $A(\Gamma)$ is constructed in 12 that contains as subalgebras both the algebra of $\Gamma$-modular forms and the Hecke ring at level $\Gamma$. There is also a corresponding action of $\mathcal{H}_{1}$ on $A(\Gamma)$.

For symmetries of type A, B, C, and D there is a corresponding crossed product, or smash product, construction that generalizes crossed products for actions of a group. We recall these constructions only for A and D symmetries, as well as a more elaborate version called bicrossed product construction. We shall see that Connes-Moscovici's Hopf algebra $\mathcal{H}_{1}$ is a bicrossed product of two, easy to describe, Hopf algebras. 
Let $A$ be a left $H$-module algebra. The underlying vector space of the crossed product algebra $A \rtimes H$ is the vector space $A \otimes H$ and its product is determined by

$$
(a \otimes g)(b \otimes h)=a\left(g^{(1)} b\right) \otimes g^{(2)} h .
$$

One checks that endowed with $1 \otimes 1$ as its unit, $A \rtimes H$ is an associative unital algebra. For example, for $H=k G$, the group algebra of a discrete group $G$ acting by automorphisms on an algebra $A$, the algebra $A \rtimes \mathcal{H}$ is isomorphic to the crossed product algebra $A \rtimes G$. For a second simple example, let a Lie algebra $\mathfrak{g}$ act via derivations on a commutative algebra $A$. Then the crossed product algebra $A \rtimes U(\mathfrak{g})$ is a subalgebra of the algebra of differential operators on $A$ generated by derivations from $\mathfrak{g}$ and multiplication operators by elements of $A$. The simples example is when $A=k[x]$ and $\mathfrak{g}=k$ acting via $\frac{d}{d x}$ on $A$. Then $A \rtimes U(\mathfrak{g})$ is the weyl algebra of differential operators on the line with polynomial coefficients.

Let $D$ be a right $H$-comodule coalgebra via the coaction $d \mapsto d^{(0)} \otimes d^{(1)} \in$ $D \otimes H$. The underlying linear space of the crossed product coalgebra $H \rtimes D$ is $H \otimes D$. It is a coalgebra whose coproduct and counit are defined by

$$
\Delta(h \otimes d)=h^{(1)} \otimes\left(d^{(1)}\right)^{(0)} \otimes h^{(2)}\left(d^{(1)}\right)^{(1)} \otimes d^{(2)}, \quad \varepsilon(h \otimes d)=\varepsilon(d) \varepsilon(h) .
$$

The above two constructions deform multiplication or comultiplication, of algebras or coalgebras, respectively. Thus to obtain a simultaneous deformation of multiplication and comultiplication of a Hopf algebra it stands to reason to try to apply both constructions simultaneously. This idea, going back to G. I. Kac in 1960's in the context of Kac-von Neumann Hopf algebras, has now found its complete generalization in the notion of bicrossed product of matched pairs of Hopf algebras. See Majid's book [26] for extensive discussions and references. There are many variations of this construction of which the most relevant for the structure of the Connes-Moscovici Hopf algebra is the following.

Let $U$ and $F$ be two Hopf algebras. We assume that $F$ is a left $U$ module algebra and $U$ is a right $F$-comodule coalgebra via $\rho: U \rightarrow U \otimes F$. We say that $(U, F)$ is a matched pair if the action and coaction satisfy the compatibility condition:

$$
\begin{gathered}
\epsilon(u(f))=\epsilon(u) \epsilon(f), \quad \Delta(u(f))=\left(u^{(1)}\right)^{(0)}\left(f^{(1)}\right) \otimes\left(u^{(1)}\right)^{(1)}\left(u^{(2)}\left(f^{(2)}\right)\right), \\
\rho(1)=1 \otimes 1, \quad \rho(u v)=\left(u^{(1)}\right)^{(0)} v^{(0)} \otimes\left(u^{(1)}\right)^{(1)}\left(u^{(2)}\left(v^{(1)}\right)\right), \\
\left(u^{(2)}\right)^{(0)} \otimes\left(u^{(1)}(f)\right)\left(u^{(2)}\right)^{(1)}=\left(u^{(1)}\right)^{(0)} \otimes\left(u^{(1)}\right)^{(1)}\left(u^{(2)}(f)\right) .
\end{gathered}
$$


Given a matched pair as above, we define its bicrossed product Hopf algebra $F \rtimes U$ to be $F \otimes U$ with crossed product algebra structure and crossed coproduct coalgebra structure. Its antipode $S$ is defined by

$$
S(f \otimes u)=\left(1 \otimes S\left(u^{(0)}\right)\right)\left(S\left(f u^{(1)}\right) \otimes 1\right) .
$$

It is a remarkable fact that, thanks to the the above compatibility conditions, all the axioms of a Hopf algebra are satisfied for $F \rtimes U$.

The simplest and first example of this bicrossed product construction is as follows. Let $G=G_{1} G_{2}$ be a factorization of a finite group $G$. This means that $G_{1}$ and $G_{2}$ are subgroups of $G, G_{1} \cap G_{2}=\{e\}$, and $G_{1} G_{2}=G$. We denote the factorization of $g$ by $g=g_{1} g_{2}$. The relation $g \cdot h:=(g h)_{2}$ for $g \in G_{1}$ and $h \in G_{2}$ defines a left action of $G_{1}$ on $G_{2}$. Similarly $g \bullet h:=(g h)_{1}$ defines a right action of $G_{2}$ on $G_{1}$. The first action turns $F=F\left(G_{2}\right)$ into a left $U=k G_{1}$-module algebra. The second action turns $U$ into a right $F$-comodule coalgebra. The later coaction is simply the dual of the map $F\left(G_{1}\right) \otimes k G_{2} \rightarrow F\left(G_{1}\right)$ induced by the right action of $G_{2}$ on $G_{1}$. Details of this example can be found in [26] and [9].

Example 2.1. 1. By a theorem of Kostant [29], any cocommutative Hopf algebra $H$ over an algebraically closed field $k$ of characteristic zero is isomorphic (as a Hopf algebra) with a crossed product algebra $H=U(P(H)) \rtimes$ $k G(H)$, where $P(H)$ is the Lie algebra of primitive elements of $H$ and $G(H)$ is the group of all grouplike elements of $H$ and $G(H)$ acts on $P(H)$ by inner automorphisms $(g, h) \mapsto g h g^{-1}$, for $g \in G(H)$ and $h \in P(H)$. The coalgebra structure of $H=U(P(H)) \rtimes k G(H)$ is simply the tensor product of the two coalgebras $U(P(H))$ and $k G(H)$.

2. We show that the Connes-Moscovici Hopf algebra is a bicrossed Hopf algebra. Let $G=\operatorname{Dif} f(\mathbb{R})$ denote the group of diffeomorphisms of the real line. It has a factorization of the form

$$
G=G_{1} G_{2},
$$

where $G_{1}$ is the subgroup of diffeomorphisms that satisfy

$$
\varphi(0)=0, \quad \varphi^{\prime}(0)=1,
$$

and $G_{2}$ is the ax $+b$ - group of affine transformations. The first Hopf algebra, $F$, is formally speaking, the algebra of polynomial functions on the 
prounipotent group $G_{1}$. It can also be defined as the "continuous dual" of the enveloping algebra of the Lie algebra of $G_{1}$. It is a commutative Hopf algebra generated by functions $\delta_{n}, n=1,2, \ldots$, defined by

$$
\delta_{n}(\varphi)=\frac{d^{n}}{d t^{n}}\left(\left.\log \left(\varphi^{\prime}(t)\right)\right|_{t=0}\right.
$$

The second Hopf algebra, $U$, is the universal enveloping algebra of the Lie algebra $\mathfrak{g}_{2}$ of the ax + b-group. It has generators $X$ and $Y$ and one relation $[X, Y]=X$.

The Hopf algebra $F$ has a right $U$-module algebra structure defined by

$$
\delta_{n}(X)=-\delta_{n+1}, \quad \text { and } \delta_{n}(Y)=-n \delta_{n} .
$$

The Hopf algebra $U$ is a left $F$-comodule coalgebra via

$$
X \mapsto 1 \otimes X+\delta_{1} \otimes X, \quad \text { and } Y \mapsto 1 \otimes Y
$$

One can check that they are a matched pair of Hopf algebras and the resulting bicrossed product Hopf algebra is isomorphic to the Connes-Moscovici Hopf algebra $\mathcal{H}_{1}$. See [9] for a slightly different approach and fine points of the proof.

3. Another important example of a bicrossed construction is the Drinfeld double $D(H)$ of a finite dimensional Hopf algebra $H$ defined as a bicrossed product $H \rtimes H^{*}[26]$.

\subsection{Modular pairs}

Let $H$ be a Hopf algebra, $\delta: H \rightarrow k$ a character and $\sigma \in H$ a grouplike element. Following [9, 10, we say that $(\delta, \sigma)$ is a modular pair if $\delta(\sigma)=1$, and a modular pair in involution if in addition we have

$$
\widetilde{S}_{\delta}^{2}=A d_{\sigma}, \quad \text { or, } \quad \widetilde{S}_{\delta}^{2}(h)=\sigma h \sigma^{-1},
$$

for all $h$ in $H$. Here the $\delta$-twisted antipode $\widetilde{S}_{\delta}: H \rightarrow H$ is defined by $\widetilde{S}_{\delta}=\delta * S$, i.e.

$$
\widetilde{S}_{\delta}(h)=\delta\left(h^{(1)}\right) S\left(h^{(2)}\right),
$$

for all $h \in H$. 
The notion of an invariant trace for actions of groups and Lie algebras can be extended to the Hopf setting. For applications to transverse geometry and number theory, it is important to formulate a notion of 'invariance' under the presence of a modular pair. Let $A$ be an $H$-module algebra, $\delta$ a character of $H$, and $\sigma \in H$ a grouplike element. A $k$-linear map $\tau: A \rightarrow k$ is called $\delta$-invariant if for all $h \in H$ and $a \in A$,

$$
\tau(h(a))=\delta(h) \tau(a)
$$

$\tau$ is called a $\sigma$-trace if for all $a, b$ in $A$,

$$
\tau(a b)=\tau(b \sigma(a))
$$

For the following lemma from [1] the fact that $A$ is unital is crucial. For $a, b \in A$, let

$$
<a, b>:=\tau(a b)
$$

Lemma 2.1. (Integration by parts formula). Let $\tau$ be a $\sigma$-trace on $A$. Then $\tau$ is $\delta$-invariant if and only if the integration by parts formula holds:

$$
<h(a), b>=<a, \widetilde{S}_{\delta}(h)(b)>
$$

for all $h \in H$ and $a, b \in A$.

Loosely speaking, the lemma says that the formal adjoint of the differential operator $h$ is $\widetilde{S}_{\delta}(h)$.

Example 2.2. 1. For any Hopf algebra $H$, the pair $(\varepsilon, 1)$ is modular. It is involutive if and only if $S^{2}=i d$. This happens, for example, when $H$ is a commutative or cocommutative Hopf algebra.

2. The original non-trivial example of a modular pair in involution is the pair $(\delta, 1)$ for Connes-Moscovici Hopf algebra $\mathcal{H}_{1}$. Let $\delta$ denote the unique extension of the modular character

$$
\delta: \mathfrak{g}_{a f f} \rightarrow \mathbb{R}, \quad \delta(X)=1, \delta(Y)=0,
$$

to a character $\delta: U\left(\mathfrak{g}_{a f f}\right) \rightarrow \mathbb{C}$. There is a unique extension of $\delta$ to a character, denoted by the same symbol $\delta: \mathcal{H}_{1} \rightarrow \mathbb{C}$. Indeed the relations $\left[Y, \delta_{n}\right]=n \delta_{n}$ show that we must have $\delta\left(\delta_{n}\right)=0$, for $n=1,2, \cdots$. One can then check that these relations are compatible with the algebra structure of 
$\mathcal{H}_{1}$. Recall the algebra $A_{\Gamma}=C_{0}^{\infty}\left(F^{+}(M) \rtimes \Gamma\right.$ from Section 1.2. It admits a $\delta$-invariant trace $\tau: A_{\Gamma} \rightarrow \mathbb{C}$ under its canonical $\mathcal{H}_{1}$ action given by [9]:

$$
\tau\left(f U_{\varphi}^{*}\right)=\int_{F^{+}(M)} f\left(y, y_{1}\right) \frac{d y d y_{1}}{y_{1}^{2}}, \quad \text { if } \varphi=1,
$$

and $\tau\left(f U_{\varphi}^{*}\right)=0$, otherwise.

3. Let $H=A\left(S L_{q}(2, k)\right)$ denote the Hopf algebra of functions on quantum $S L(2, k)$. As an algebra it is generated by symbols $x, u, v, y$, with the following relations:

$$
\begin{gathered}
u x=q x u, \quad v x=q x v, \quad y u=q u y, \quad y v=q v y, \\
u v=v u, \quad x y-q^{-1} u v=y x-q u v=1 .
\end{gathered}
$$

The coproduct, counit and antipode of $\mathcal{H}$ are defined by

$$
\begin{gathered}
\Delta(x)=x \otimes x+u \otimes v, \quad \Delta(u)=x \otimes u+u \otimes y, \\
\Delta(v)=v \otimes x+y \otimes v, \quad \Delta(y)=v \otimes u+y \otimes y, \\
\epsilon(x)=\epsilon(y)=1, \quad \epsilon(u)=\epsilon(v)=0, \\
S(x)=y, \quad S(y)=x, \quad S(u)=-q u, \quad S(v)=-q^{-1} v .
\end{gathered}
$$

Define a character $\delta: H \rightarrow k$ by:

$$
\delta(x)=q, \quad \delta(u)=0, \quad \delta(v)=0, \quad \delta(y)=q^{-1} .
$$

One checks that $\widetilde{S}_{\delta}^{2}=i d$. This shows that $(\delta, 1)$ is a modular pair for $H$. This example and its Hopf-cyclic cohomology is studied in [20].

More generally, it is shown in [10] that coribbon Hopf algebras and compact quantum groups are endowed with canonical modular pairs of the form $(\delta, 1)$ and, dually, ribbon Hopf algebras have canonical modular pairs of the type $(1, \sigma)$.

4. We will see in the next section that modular pairs in involution are in fact one dimensional cases of what we call stable anti-Yetter-Drinfeld modules, i.e. they are one dimensional noncommutative local systems that one can introduce into Hopf algebra equivariant cyclic cohomology. 


\section{Anti-Yetter-Drinfeld modules}

An important question left open in our paper [21] was the issue of identifying the most general class of coefficients allowable in cyclic (co)homology of Hopf algebras and Hopf-cyclic cohomology in general. This problem is completely solved, among other things, in 14. It is shown in this paper that the most general coefficients are the class of so called stable anti-Yetter-Drinfeld modules. In Section 3.2 we briefly report on this very recent development as well.

It is quite surprising that when the general formalism of cyclic cohomology theory, namely the theory of (co)cyclic modules [3], is applied to Hopf algebras, variations of such standard notions like Yetter-Drinfeld (YD) modules appear naturally. The so called anti-Yetter-Drinfeld modules introduced in 14 are twistings, by modular pairs in involution, of YD modules. This means that the category of anti-Yetter-Drinfeld modules is a "mirror image" of the category of YD modules. We mention that anti-Yetter-Drinfeld modules were considered independently also by $\mathrm{C}$. Voigt in connection with his work on equivariant cyclic cohomology [31].

\subsection{Yetter-Drinfeld modules}

Yetter-Drinfeld modules were introduced by D. Yetter under the name crossed bimodules [32. The present name was coined in [27]. One of the motivations was to define a braiding on the monoidal category $H-M o d$ of representations of a, not necessarily commutative or cocommutative, Hopf algebra $H$. To define such a braiding one should either restrict to special classes of Hopf algebras, or, to special classes of modules. Drinfeld showed that when $H$ is a quasitriangular Hopf algebra, then $H-M o d$ is a braided monoidal category. See 26] for definitions and references. Dually, when $H$ is coquasitriangular, the category $H-C o m o d$ of $H$-comodules is a braided monoidal category. In 32 Yetter shows that to obtain a braiding on a subcategory of $H-M o d$, for an arbitrary $H$, one has essentially one choice and that is restricting to the class of Yetter-Drinfeld modules as we explain now.

Let $H$ be a Hopf algebra and $M$ be a left $H$-module and left $H$-comodule simultaneously. We say that $M$ is a left-left Yetter-Drinfeld $H$-module if the two structures on $M$ are compatible in the sense that

$$
\rho(h m)=h^{(1)} m^{(-1)} S\left(h^{(3)}\right) \otimes h^{(2)} m^{(0)},
$$


for all $h \in H$ and $m \in M$ [26, 27, 32]. One can similarly define left-right, right-left and right-right YD modules. A morphism of YD modules $M \rightarrow N$ is an $H$-linear and $H$-colinear map $f: M \rightarrow N$. We denote the category of left-left YD modules over $H$ by ${ }_{H}^{H} \mathcal{Y} \mathcal{D}$.

This notion is closely related to the Drinfeld double of finite dimensional Hopf algebras. In fact if $H$ is finite dimensional, then one can show that the category ${ }_{H}^{H} \mathcal{Y} \mathcal{D}$ is isomorphic to the category of left modules over the Drinfeld double $D(H)$ of $H$ 26].

The following facts about the category ${ }_{H}^{H} \mathcal{Y} \mathcal{D}$ are well known [26, 27, 32]:

1. The tensor product $M \otimes N$ of two YD modules is a YD module. Its module and comodule structure are the standard ones:

$$
h(m \otimes n)=h^{(1)} m \otimes h^{(1)} n, \quad(m \otimes n) \mapsto m^{(-1)} n^{(-1)} \otimes m^{(0)} \otimes n^{(0)} .
$$

This shows that the category ${ }_{H}^{H} \mathcal{Y} \mathcal{D}$ is a monoidal subcategory of the monoidal category $H-M o d$.

2. The category ${ }_{H}^{H} \mathcal{Y} \mathcal{D}$ is a braided monoidal category under the braiding

$$
c_{M, N}: M \otimes N \rightarrow N \otimes M, \quad m \otimes n \mapsto m^{(-1)} \cdot n \otimes m^{(0)} .
$$

In fact Yetter proves a strong inverse to this statement as well [32] for any small strict monoidal category $\mathcal{C}$ endowed with a monoidal functor $F: \mathcal{C} \rightarrow$ Vect $_{f}$ to the category of finite dimensional vector spaces, there is a Hopf algebra $H$ and a monoidal functor $\tilde{F}: \mathcal{C} \rightarrow_{H}^{H} \mathcal{Y} \mathcal{D}$ such that the following diagram comutes

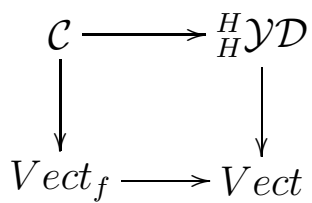

3. The category ${ }_{H}^{H} \mathcal{Y} \mathcal{D}$ is the center of the monoidal category $H-M o d$. Recall that the (left) center $\mathcal{Z C}$ of a monoidal category [18] is a category whose objects are pairs $\left(X, \sigma_{X,-}\right)$, where $X$ is an object of $\mathcal{C}$ and $\sigma_{X,-}: X \otimes-\rightarrow$ $-\otimes X$ is a natural isomorphism satisfying certain compatibility axioms with associativity and unit constraints. It can be shown that the center of a monoidal category is a braided monoidal category and $\mathcal{Z}(H-M o d)={ }_{H}^{H} \mathcal{Y} \mathcal{D}$ [18]. 
Example 3.1. 1. Let $H=k G$ be the group algebra of a discrete group $G$. $A$ left $k G$-comodule is simply a $G$-graded vector space

$$
M=\bigoplus_{g \in G} M_{g}
$$

where the coaction is defined by

$$
m \in M_{g} \mapsto g \otimes m
$$

An action of $G$ on $M$ defines a $Y D$ module structure iff for all $g, h \in G$,

$$
h m \in M_{h g h^{-1}}
$$

This example can be explained as follows. Let $\mathcal{G}$ be a groupoid whose objects are $G$ and its morphisms are defined by

$$
\operatorname{Hom}(g, h)=\left\{k \in G ; k g k^{-1}=h\right\} .
$$

Recall that an action of a groupoid $\mathcal{G}$ on the category Vect of vector spaces is simply a functor $F: \mathcal{G} \rightarrow$ Vect. Then it is easily seen that we have a one-one correspondence between $Y D$ modules for $k G$ and actions of $\mathcal{G}$ on Vect. This example clearly shows that one can think of an $Y D$ module over $k G$ as an 'equivariant sheaf' on $G$ and of $Y D$ modules as noncommutative analogues of equivariant sheaves on a topological group.

2. If $H$ is cocommutative then any left $H$-module $M$ can be turned into a left-left $Y D$ module via the coaction $m \mapsto 1 \otimes m$. Similarly, when $H$ is cocmmuntative then any left $H$-comodule $M$ can be turned into a YD module via the $H$-action $h \cdot m:=\varepsilon(h) m$.

3. Any Hopf algebra acts on itself via conjugation action $g \cdot h:=g^{(1)} h S\left(g^{(2)}\right)$ and coacts via translation coaction $h \mapsto h^{(1)} \otimes h^{(2)}$. It can be checked that this endows $M=H$ with a YD module structure.

\subsection{Stable anti-Yetter-Drinfeld modules}

This class of modules for Hopf algebras were introduced for the first time in [14. Unlike Yetter-Drinfeld modules, its definition, however, was entirely motivated and dictated by cyclic cohomology theory: the anti-Yetter-Drinfeld 
condition guarantees that the simplicial and cyclic operators are well defined on invariant complexes and the stability condition implies that the crucial periodicity condition for cyclic modules are satisfied.

Definition 3.1. A left-left anti-Yetter-Drinfeld $H$-module is a left $H$-module and left $H$-comodule such that

$$
\rho(h m)=h^{(1)} m^{(-1)} S\left(h^{(3)}\right) \otimes h^{(2)} m^{(0)},
$$

for all $h \in H$ and $m \in M$. We say that $M$ is stable if in addition we have

$$
m^{(-1)} m^{(0)}=m
$$

for all $m \in M$.

There are of course analogous definitions for left-right, right-left and rightright stable anti-Yetter-Drinfeld (SAYD) modules. We note that by changing $S$ to $S^{-1}$ in the above equation, we obtain the compatibility condition for a Yetter-Drinfeld module from the previous subsection.

The following lemma from [14 shows that 1-dimensional SAYD modules correspond to Connes-Moscovici's modular pairs in involution:

Lemma 3.1. There is a one-one correspondence between modular pairs in involution $(\delta, \sigma)$ on $H$ and $S A Y D$ module structure on $M=k$, defined by

$$
h . r=\delta(h) r, \quad r \mapsto \sigma \otimes r,
$$

for all $h \in H$ and $r \in k$. We denote this module by $M={ }^{\sigma} k_{\delta}$.

Let ${ }_{H}^{H} \mathcal{A Y \mathcal { D }}$ denote the category of left-left anti-Yetter-Drinfeld $H$-modules, where morphisms are $H$-linear and $H$-colinear maps. Unlike YD modules, anti-Yetter-Drinfeld modules do not form a monoidal category under the standard tensor product. This can be checked easily on 1-dimensional modules given by modular pairs in involution. The following result of [14], however, shows that the tensor product of an anti-Yetter-Drinfeld module with a Yetter-Drinfeld module is again anti-Yetter-Drinfeld.

Lemma 3.2. Let $M$ be a Yetter-Drinfeld module and $N$ be an anti-YetterDrinfeld module (both left-left). Then $M \otimes N$ is an anti-Yetter-Drinfeld module under the diagonal action and coaction:

$$
h(m \otimes n)=h^{(1)} m \otimes h^{(1)} n, \quad(m \otimes n) \mapsto m^{(-1)} n^{(-1)} \otimes m^{(0)} \otimes n^{(0)} .
$$


In particular, using a modular pair in involution $(\delta, \sigma)$, we obtain a functor

$$
{ }_{H}^{H} \mathcal{Y D} \rightarrow{ }_{H}^{H} \mathcal{A} \mathcal{Y D}, \quad M \mapsto \bar{M}={ }^{\circ} \otimes M
$$

This result clearly shows that AYD modules can be regarded as the twisted analogue or mirror image of YD modules, with twistings provided by modular pairs in involution. This result was later strengthened by the following result, pointed out to us by M. Staic [28]. It shows that if the Hopf algebra has a modular pair in involution then the category of YD modules is equivalent to the category of AYD modules:

Proposition 3.1. Let $H$ be a Hopf algebra, $(\delta, \sigma)$ a modular pair in involution and $M$ an anti-Yetter-Drinfeld module. If we define $m \cdot h=m h^{(1)} \delta\left(S\left(h^{(2)}\right)\right)$ and $\rho(m)=\sigma^{-1} m^{(-1)} \otimes m^{(0)}$, then $(M, \cdot, \rho)$ is an Yetter-Drinfeld module. Moreover in this way we get an isomorphism between the categories of $A Y D$ modules and YD mpdules.

It follows that tensoring with ${ }^{\sigma^{-}} k_{\delta \circ S}$ turns the anti-Yetter-Drinfeld modules to Yetter-Drinfeld modules and this is the inverse for the operation of tensoring with $\hbar_{\delta}$.

Example 3.2. 1. For Hopf algebras with $S^{2}=I$, e.g. commutative or cocommutative Hopf algebras, there is no distinction between $Y D$ and $A Y D$ modules. This applies in particular to $H=k G$ and Example 2.1.1. The stability condition $m^{(-1)} m^{(0)}=m$ is equivalent to

$$
g \cdot m=m, \quad \text { for all } g \in G, m \in M_{g} \text {. }
$$

2. Hopf-Galois extensions are noncommutative analogues of principal bundles in (affine) algebraic geometry. Following [14] we show that they give rise to large classes of examples of SAYD modules. Let $P$ be a right $H$-comodule algebra, and let

$$
B:=P^{H}=\{p \in P ; \rho(p)=p \otimes 1\}
$$

be the space of coinvariants of $P$. It is easy to see that $B$ is a subalgebra of $P$. The extension $B \subset P$ is called a Hopf-Galois extension if the map

$$
\text { can }: P \otimes_{B} P \rightarrow B \otimes H, \quad p \otimes p^{\prime} \mapsto p \rho\left(p^{\prime}\right),
$$


is bijective. (Note that in the commutative case this corresponds to the condition that the action of the structure group on fibres is free). The bijectivity assumption allows us to define the translation map $T: H \rightarrow P \otimes_{B} P$,

$$
T(h)=\operatorname{can}^{-1}(1 \otimes h)=h^{(\overline{1})} \otimes h^{(\overline{2})} .
$$

It can be checked that the centralizer $Z_{B}(P)=\{p \mid b p=p b \quad \forall b \in B\}$ of $B$ in $P$ is a subcomodule of $P$. There is an action of $H$ on $Z_{B}(P)$ defined by $p h=h^{(1)} p h^{(2)}$ called the Miyashita-Ulbrich action. It is shown that this action and coaction satisfy the Yetter-Drinfeld compatibility condition. On the other hand if $B$ is central, then by defining the new action $p h=\left(S^{-1}(h)\right)^{(2)} p\left(S^{-1}(h)\right)^{(1)}$ and the right coaction of $P$ we have a $S A Y D$ module. This example was the starting point of [17] where the relative cyclic homology of Hopf-Galois extensions is related to a variant of Hopf-cyclic cohomology with coefficients in stable anti-Yetter-Drinfeld modules. In [23] we showed that the theory introduced in [17] is ismorphic to one of the theories introduced in [15].

3. Let $M=H$. Then with conjugation action $g \cdot h=g^{(1)} h S\left(g^{(2)}\right)$ and comultiplication $h \mapsto h^{(1)} \otimes h^{(2)}$ as coaction, $M$ is an SAYD module.

\section{Hopf-cyclic cohomology}

In this section we first recall the approach by Connes and Moscovici towards the definition of their cyclic cohomology theory for Hopf algebras. The characteristic map $\chi_{\tau}$ palys an imporant role here. Then we switch to the point of view adopted in [21] based on invariant complexes, culminating in our joint work [14, 15]. The resulting Hopf-cyclic cohomology theories of type $A, B$, and $C$, and their corresponding cyclic modules, contain all known examples of cyclic theory discovered so far. We note that very recently A. Kaygun has extended the Hopf-cyclic cohomology to a cohomology for bialgebras with coefficients in stable modules. For Hopf algebras it reduces to Hopf-cyclic cohomology [19].

\subsection{Connes-Moscovici's breakthrough}

Without going into details, in this section we formulate one of the problems that was faced and solved by Connes and Moscovici in the course of their 
study of an index problem on foliated manifolds [9]. See also [11] for a survey. As we shall see, this led them to a new cohomology theory for Hopf algebras that is now an important example of Hopf-cyclic cohomology.

The local index formula of Connes and Moscovici 8 gives the Chern character $C h(A, h, D)$ of a regular spectral triple $(A, h, D)$ as a cyclic cocycle in the $(b, B)$-bicomplex of the algebra $A$. For spectral triples of interest in transverse geometry [9], this cocycle is differentiable in the sense that it is in the image of the Connes-Moscovici characteristic map $\chi_{\tau}$ defined below, with $H=\mathcal{H}_{1}$ and $A=\mathcal{A}_{\Gamma}$. To identify this class in terms of characteristic classes of foliations, it would be extremely helpful to show that it is the image of a cocycle for a cohomology theory for Hopf algebras. This is rather similar to the situation for classical characteristic classes which are pull backs of group cohomology classes.

We can formulate this problem abstractly as follows: Let $H$ be a Hopf algebra endowed with a modular pair in involution $(\delta, \sigma)$, and $A$ be an $H$ module algebra. Let $\tau: A \rightarrow k$ be a $\delta$-invariant $\sigma$-trace on $A$ as defined in Section 1.3. Consider the Connes-Moscovici characteristic map

$$
\begin{gathered}
\chi_{\tau}: H^{\otimes n} \longrightarrow \operatorname{Hom}\left(A^{\otimes(n+1)}, k\right), \\
\chi_{\tau}\left(h_{1} \otimes \cdots \otimes h_{n}\right)\left(a_{0} \otimes \cdots \otimes a_{n}\right)=\tau\left(a_{0} h_{1}\left(a_{1}\right) \cdots h_{n}\left(a_{n}\right)\right) .
\end{gathered}
$$

Now the burning question is: can we promote the collection of spaces $\left\{H^{\otimes n}\right\}_{n \geq 0}$ to a cocyclic module such that the characteristic map $\chi_{\tau}$ turns into a morphism of cocyclic modules? We recall that the face, degeneracy, and cyclic operators for $\operatorname{Hom}\left(A^{\otimes(n+1)}, k\right)$ are defined by [3]:

$$
\begin{aligned}
\delta_{i}^{n} \varphi\left(a_{0}, \cdots, a_{n+1}\right) & =\varphi\left(a_{0}, \cdots, a_{i} a_{i+1}, \cdots, a_{n+1}\right), \quad i=0, \cdots, n, \\
\delta_{n+1}^{n} \varphi\left(a_{0}, \cdots, a_{n+1}\right) & =\varphi\left(a_{n+1} a_{0}, a_{1}, \cdots, a_{n}\right), \\
\sigma_{i}^{n} \varphi\left(a_{0}, \cdots, a_{n}\right) & =\varphi\left(a_{0}, \cdots, a_{i}, 1, \cdots, a_{n}\right), \quad i=0, \cdots, n \\
\tau_{n} \varphi\left(a_{0}, \cdots, a_{n}\right) & =\varphi\left(a_{n}, a_{0}, \cdots, a_{n-1}\right) .
\end{aligned}
$$

The relation

$$
h(a b)=h^{(1)}(a) h^{(2)}(b)
$$

shows that in order for $\chi_{\tau}$ to be compatible with face operators, the face operators on $H^{\otimes n}$ must involve the coproduct of $H$. In fact if we define, for 


$$
\begin{aligned}
0 \leq i \leq n, \delta_{i}^{n}: H^{\otimes n} \rightarrow H^{\otimes(n+1)} & \text { by } \\
\delta_{0}^{n}\left(h_{1} \otimes \cdots \otimes h_{n}\right) & =1 \otimes h_{1} \otimes \cdots \otimes h_{n}, \\
\delta_{i}^{n}\left(h_{1} \otimes \cdots \otimes h_{n}\right) & =h_{1} \otimes \cdots \otimes h_{i}^{(1)} \otimes h_{i}^{(2)} \otimes \cdots \otimes h_{n}, \\
\delta_{n+1}^{n}\left(h_{1} \otimes \cdots \otimes h_{n}\right) & =h_{1} \otimes \cdots \otimes h_{n} \otimes \sigma,
\end{aligned}
$$

then we have, for all $n$ and $i$,

$$
\chi_{\tau} \delta_{i}^{n}=\delta_{i}^{n} \chi_{\tau}
$$

Similarly, the relation $h\left(1_{A}\right)=\varepsilon(h) 1_{A}$, shows that the degeneracy operators on $H^{\otimes n}$ should involve the counit of $H$. We thus define

$$
\sigma_{i}^{n}\left(h_{1} \otimes \cdots \otimes h_{n}\right)=h_{1} \otimes \cdots \otimes \varepsilon\left(h_{i}\right) \otimes \cdots \otimes h_{n} .
$$

The most difficult part in this regard is to guess the form of the cyclic operator $\tau_{n}: H^{\otimes n} \rightarrow H^{\otimes n}$. Compatibility with $\chi_{\tau}$ demands that

$$
\tau\left(a_{0} \tau_{n}\left(h_{1} \otimes \cdots \otimes h_{n}\right)\left(a_{1} \otimes \cdots \otimes a_{n}\right)\right)=\tau\left(a_{n} h_{1}\left(a_{0}\right) h_{2}\left(a_{1}\right) \cdots h_{n}\left(a_{n-1}\right)\right),
$$

for all $a_{i}$ 's and $h_{i}$ 's. Now integration by parts formula in Lemma 2.1, combined with the $\sigma$-trace property of $\tau$, gives us:

$$
\tau\left(a_{1} h\left(a_{0}\right)\right)=\tau\left(h\left(a_{0}\right) \sigma\left(a_{1}\right)\right)=\tau\left(a_{0} \tilde{S}_{\delta}(h)\left(\sigma\left(a_{1}\right)\right) .\right.
$$

This suggests that we should define $\tau_{1}: H \rightarrow H$ by

$$
\tau_{1}(h)=\tilde{S}_{\delta}(h) \sigma .
$$

Note that the condition $\tau_{1}^{2}=I$ is equivalent to the involutive condition $\tilde{S}_{\delta}^{2}=A d_{\sigma}$.

For any $n$, integration by parts formula together with the $\sigma$-trace property shows that:

$$
\begin{aligned}
\tau\left(a_{n} h_{1}\left(a_{0}\right) \cdots h_{n}\left(a_{n-1}\right)\right) & =\tau\left(h_{1}\left(a_{0}\right) \cdots h_{n}\left(a_{n-1}\right) \sigma\left(a_{n}\right)\right) \\
& =\tau\left(a_{0} \tilde{S}_{\delta}\left(h_{1}\right)\left(h_{2}\left(a_{1}\right) \cdots h_{n}\left(a_{n-1}\right) \sigma\left(a_{n}\right)\right)\right) \\
& =\tau\left(a_{0} \tilde{S}_{\delta}\left(h_{1}\right) \cdot\left(h_{2} \otimes \cdots \otimes h_{n} \otimes \sigma\right)\left(a_{1} \otimes \cdots \otimes a_{n}\right) .\right.
\end{aligned}
$$

This suggests that the Hopf-cyclic operator $\tau_{n}: H^{\otimes n} \rightarrow H^{\otimes n}$ should be defined as

$$
\tau_{n}\left(h_{1} \otimes \cdots \otimes h_{n}\right)=\tilde{S}_{\delta}\left(h_{1}\right) \cdot\left(h_{2} \otimes \cdots \otimes h_{n} \otimes \sigma\right)
$$


where $\cdot$ denotes the diagonal action defined by

$$
h \cdot\left(h_{1} \otimes \cdots \otimes h_{n}\right):=h^{(1)} h_{1} \otimes h^{(2)} h_{2} \otimes \cdots \otimes h^{(n)} h_{n} .
$$

We let $\tau_{0}=I: H^{\otimes 0}=k \rightarrow H^{\otimes 0}$, be the identity map. The remarkable fact, proved by Connes and Moscovici [9, 10, is that endowed with the above face, degeneracy, and cyclic operators, $\left\{H^{\otimes n}\right\}_{n \geq 0}$ is a cocyclic module. The resulting cyclic cohomology groups are denoted by $H C_{(\delta, \sigma)}^{n}(H), n=0,1, \cdots$ and we obtain the desired characteristic map

$$
\chi_{\tau}: H C_{(\delta, \sigma)}^{n}(H) \rightarrow H C^{n}(A) .
$$

As with any cocyclic module, cyclic cohomology can also be defined in terms of cyclic cocycles. In this case a cyclic n-cocycle is an element $x \in H^{\otimes n}$ satisfying the conditions

$$
b x=0, \quad(1-\lambda) x=0,
$$

where $b: H^{\otimes n} \rightarrow H^{\otimes(n+1)}$ and $\lambda: H^{\otimes n} \rightarrow H^{\otimes n}$ are defined by

$$
\begin{aligned}
b\left(h^{1} \otimes \cdots \otimes h^{n}\right) & =1 \otimes h_{1} \otimes \cdots \otimes h_{n} \\
& +\sum_{i=1}^{n}(-1)^{i} h_{1} \otimes \cdots \otimes h_{i}^{(1)} \otimes h_{i}^{(2)} \otimes \cdots \otimes h_{n} \\
& +(-1)^{n+1} h_{1} \otimes \cdots \otimes h_{n} \otimes \sigma, \\
\lambda\left(h_{1} \otimes \cdots \otimes h_{n}\right) & =(-1)^{n} \tilde{S}_{\delta}\left(h_{1}\right) \cdot\left(h_{2} \otimes \cdots \otimes h_{n} \otimes \sigma\right) .
\end{aligned}
$$

The cyclic cohomology groups $H C_{(\delta, \sigma)}^{n}(H)$ are computed in several cases in 9]. Of particular interest for applications to transverse index theory and number theory is the the (periodic) cyclic cohomology of the ConnesMoscovici Hopf algebra $\mathcal{H}_{1}$. It is shown in 9] that the periodic groups $H P_{(\delta, 1)}^{n}\left(\mathcal{H}_{1}\right)$ are canonically isomorphic to the Gelfand-Fuchs cohomology of the Lie algebra of formal vector fields on the line:

$$
H^{*}\left(\mathfrak{a}_{1}, \mathbb{C}\right)=H P_{(\delta, 1)}^{*}\left(\mathcal{H}_{1}\right) .
$$

Calculation of the unstable groups is an interesting open problem. The following interesting elements are however already been identified. It can be directly checked that the elements $\delta_{2}^{\prime}:=\delta_{2}-\frac{1}{2} \delta_{1}^{2}$ and $\delta_{1}$ are cyclic 1-cocycles on $\mathcal{H}_{1}$, and

$$
F:=X \otimes Y-Y \otimes X-\delta_{1} Y \otimes Y
$$

is a cyclic 2-cocycle. See 12 for detailed calculations and relations between these cocycles and the Schwarzian derivative, Godbillon-Vey cocycle, and the transverse fundamental class of Connes [5], respectively. 


\subsection{Hopf-cyclic cohomology: type A, B, and C theo- ries}

We recall the definitions of the three cyclic cohomology theories that were defined in [15]. We call them $A, B$ and $C$ theories. In the first case the algebra $A$ is endowed with an action of a Hopf algebra; in the second case the algebra $B$ is equipped with a coaction of a Hopf algebra; and finally in theories of type $C$, we have a coalgebra endowed with an action of a Hopf algebra. In all three theories the module of coefficients is a stable anti-Yetter-Drinfeld (SAYD) module over the Hopf algebra and we attach a cocyclic module in the sense of Connes 3 to the given data. Along the same lines one can define a Hopfcyclic cohomology theory for comodule coalgebras as well (type D theory). Since so far we have found no applications of such a theory we won't give its definition here. We also show that all known examples of cyclic cohomology theories that are introduced so far such as: ordinary cyclic cohomology for algebras, Connes-Moscovici's cyclic cohomology for Hopf algebras [8], and equivariant cyclic cohomology [1, 2], are special cases of these theories.

Let $A$ be a left $H$-module algebra and $M$ be a left-right SAYD $H$-module. Then the spaces $M \otimes A^{\otimes(n+1)}$ are right $H$-modules via the diagonal action

$$
(m \otimes \widetilde{a}) h:=m h^{(1)} \otimes S\left(h^{(2)}\right) \widetilde{a},
$$

where the left $H$-action on $\widetilde{a} \in A^{\otimes(n+1)}$ is via the left diagonal action of $H$.

We define the space of equivariant cochains on $A$ with coefficients in $M$ by

$$
\mathcal{C}_{H}^{n}(A, M):=\operatorname{Hom}_{H}\left(M \otimes A^{\otimes(n+1)}, k\right) .
$$

More explicitly, $f: M \otimes A^{\otimes(n+1)} \rightarrow k$ is in $\mathcal{C}_{H}^{n}(A, M)$, if and only if

$$
f\left(\left(m \otimes a_{0} \otimes \cdots \otimes a_{n}\right) h\right)=\varepsilon(h) f\left(m \otimes a_{0} \otimes \cdots \otimes a_{n}\right),
$$

for all $h \in H, m \in M$, and $a_{i} \in A$. It is shown in [15] that the following operators define a cocyclic module structure on $\left\{\mathcal{C}_{H}^{n}(A, M)\right\}_{n \in \mathbb{N}}$ :

$$
\begin{aligned}
& \left(\delta_{i} f\right)\left(m \otimes a_{0} \otimes \cdots \otimes a_{n}\right)=f\left(m \otimes a_{0} \otimes \cdots \otimes a_{i} a_{i+1} \otimes \cdots \otimes a_{n}\right), \quad 0 \leq i<n, \\
& \left(\delta_{n} f\right)\left(m \otimes a_{0} \otimes \cdots \otimes a_{n}\right)=f\left(m^{(0)} \otimes\left(S^{-1}\left(m^{(-1)}\right) a_{n}\right) a_{0} \otimes a_{1} \otimes \cdots \otimes a_{n-1}\right), \\
& \left(\sigma_{i} f\right)\left(m \otimes a_{0} \otimes \cdots \otimes a_{n}\right)=f\left(m \otimes a_{0} \otimes \cdots \otimes a_{i} \otimes 1 \otimes \cdots \otimes a_{n}\right), \quad 0 \leq i \leq n, \\
& \left(\tau_{n} f\right)\left(m \otimes a_{0} \otimes \cdots \otimes a_{n}\right)=f\left(m^{(0)} \otimes S^{-1}\left(m^{(-1)}\right) a_{n} \otimes a_{0} \otimes \cdots \otimes a_{n-1}\right) .
\end{aligned}
$$

We denote the resulting cyclic cohomology theory by $H C_{H}^{n}(A, M), n=$ $0,1, \cdots$. 
Example 4.1. 1. For $H=k=M$ we obviously recover the standard cocyclic module of the algebra $A$. The resulting cyclic cohomology theory is the ordinary cyclic cohomology of algebras.

2. For $M=H$ and $H$ acting on $M$ by conjugation and coacting via coproduct (Example 2.2.3.), we obtain the equivariant cyclic cohomology theory of Akbarpour and Khalkhali For $H$-module algebras [1, [2].

3. For $H=k\left[\sigma, \sigma^{-1}\right]$ the Hopf algebra of Laurent polynomials, where $\sigma$ acts by automorphisms on an algebra $A$, and $M=k$ is a trivial module, we obtain the so called twisted cyclic cohomology of $A$ with respect to $\sigma$. A twisted cyclic $n$-cocycle is a linear map $f: A^{\otimes(n+1)} \rightarrow k$ satisfying:

$$
f\left(\sigma a_{n}, a_{0}, \cdots, a_{n-1}\right)=(-1)^{n} f\left(a_{0}, \cdots, a_{n}\right), \quad b_{\sigma} f=0,
$$

where $b_{\sigma}$ is the twisted Hochschild boundary defined by

$$
\begin{aligned}
b_{\sigma} f\left(a_{0}, \cdots, a_{n+1}\right) & =\sum_{i=0}^{n}(-1)^{i} f\left(a_{0}, \cdots, a_{i} a_{i+1}, \cdots, a_{n+1}\right) \\
& +(-1)^{n+1} f\left(\sigma\left(a_{n+1}\right) a_{0}, a_{1}, \cdots, a_{n}\right) .
\end{aligned}
$$

4. It is easy to see that for $M={ }^{\sigma} k_{\delta}$, the $S A Y D$ module attached to a modular pair in involution $(\delta, \sigma), H C_{H}^{0}(A, M)$ is the space of $\delta$-invariant $\sigma$-traces on $A$ in the sense of Connes-Moscovici [9, 10] (cf. Section 1.3.).

Next, let $B$ be a right $H$-comodule algebra and $M$ be a right-right SAYD $H$-module. Let

$$
\mathcal{C}^{n, H}(B, M):=\operatorname{Hom}^{H}\left(B^{\otimes(n+1)}, M\right),
$$

denote the space of right $H$-colinear $(n+1)$-linear functionals on $B$ with values in $M$. Here $B^{\otimes(n+1)}$ is considered a right $H$-comodule via the diagonal coaction of $H$ :

$$
b_{0} \otimes \cdots \otimes b_{n} \mapsto\left(b_{0}^{(0)} \otimes \cdots \otimes b_{n}^{(0)}\right) \otimes\left(b_{0}^{(1)} b_{1}^{(1)} \cdots b_{n}^{(1)}\right) .
$$

It is shown in [15] that, thanks to the invariance property imposed on our cochains and the SAYD condition on $M$, the following maps define a cocyclic 
module structure on $\left\{\mathcal{C}^{n, H}(B, M)\right\}_{n \in \mathbb{N}}$ :

$$
\begin{aligned}
& \left(\delta_{i} f\right)\left(b_{0}, \cdots, b_{n+1}\right)=f\left(b_{0}, \cdots, b_{i} b_{i+1}, \cdots, b_{n+1}\right), \quad 0 \leq i<n, \\
& \left(\delta_{n} f\right)\left(b_{0}, \cdots, b_{n+1}\right)=f\left(b_{n+1}^{(0)} b_{0}, b_{1}, \cdots, b_{n}\right) b_{n+1}^{(1)}, \\
& \left(\sigma_{i} f\right)\left(b_{0}, \cdots, b_{n-1}\right)=f\left(b_{0}, \cdots, b_{i}, 1, \cdots b_{n-1}\right), \quad 0 \leq i<n-1, \\
& \left(\tau_{n} f\right)\left(b_{0}, \cdots, b_{n}\right)=f\left(b_{n}^{(0)}, b_{0}, \cdots, b_{n-1}\right) b_{n}^{(1)} .
\end{aligned}
$$

We denote the resulting cyclic cohomology groups by $H C^{n, H}(B, M), n=$ $0,1, \cdots$.

Example 4.2. 1. For $B=H$, equipped with comultiplication as coaction, and $M={ }^{\sigma} k_{\delta}$ associated to a modular pair in involution, we obtain the Hopfcyclic cohomology of Hopf algebras as defined in [20]. This theory is different from Connes-Moscovici's theory for Hopf algebras. It is dual, in the sense of Hopf algebras and not Hom dual, to Connes-Moscovici's theory [22]. It is computed in the following cases [20]: $H=k G, H=U(\mathfrak{g})$, where it is isomorphic to group cohomology and Lie algebra cohomology, respectively; $H=S L_{2}(q, k)$, and $H=U_{q}\left(s l_{2}\right)$.

2. For $H=k$, and $M=k$ a trivial module, we obviously recover the cyclic cohomology of the algebra $B$.

Finally we describe theories of type $C$ and their main examples. As we shall see, Connes-Moscovici's original example of Hopf-cyclic cohomology belong to this class of theories. Let $C$ be a left $H$-module coalgebra, and $M$ be a right-left SAYD $H$-module. Let

$$
\mathcal{C}^{n}(C, M):=M \otimes_{H} C^{\otimes(n+1)} \quad n \in \mathbb{N} .
$$

It can be checked that, thanks to the SAYD condition on $M$, the following operators are well defined and define a cocyclic module, denoted $\left\{\mathcal{C}^{n}(C, M)\right\}_{n \in \mathbb{N}}$. In particular the crucial periodicity conditions $\tau_{n}^{n+1}=i d, n=0,1,2 \cdots$, are satisfied [15]:

$$
\begin{aligned}
\delta_{i}\left(m \otimes c_{0} \otimes \cdots \otimes c_{n-1}\right) & =m \otimes c_{0} \otimes \cdots \otimes c_{i}^{(1)} \otimes c_{i}^{(2)} \otimes c_{n-1}, 0 \leq i<n, \\
\delta_{n}\left(m \otimes c_{0} \otimes \cdots \otimes c_{n-1}\right) & =m^{(0)} \otimes c_{0}^{(2)} \otimes c_{1} \otimes \cdots \otimes c_{n-1} \otimes m^{(-1)} c_{0}^{(1)}, \\
\sigma_{i}\left(m \otimes c_{0} \otimes \cdots \otimes c_{n+1}\right) & =m \otimes c_{0} \otimes \cdots \otimes \varepsilon\left(c_{i+1}\right) \otimes \cdots \otimes c_{n+1}, 0 \leq i \leq n, \\
\tau_{n}\left(m \otimes c_{0} \otimes \cdots \otimes c_{n}\right) & =m^{(0)} \otimes c_{1} \otimes \cdots \otimes c_{n} \otimes m^{(-1)} c_{0} .
\end{aligned}
$$


Example 4.3. 1. For $H=k=M$, we recover the cocyclic module of a coalgebra which defines its cyclic cohomology.

2. For $C=H$ and $M={ }^{\sigma} k_{\delta}$, the cocyclic module $\left\{\mathcal{C}_{H}^{n}(C, M)\right\}_{n \in \mathbb{N}}$ is isomorphic to the cocyclic module of Connes-Moscovici [9], attached to a Hopf algebra endowed with a modular pair in involution. This example is truly fundamental and started the whole theory.

\section{References}

[1] R. Akbarpour, and M. Khalkhali, Hopf algebra equivariant cyclic homology and cyclic homology of crossed product algebras. J. Reine Angew. Math., 559 (2003), 137-152.

[2] R. Akbarpour, and M. Khalkhali, Equivariant cyclic cohomology of $H$ algebras. K-Theory 29 (2003), no. 4, 231-252.

[3] A. Connes, Cohomologie cyclique et foncteurs Ext ${ }^{n}$. (French) (Cyclic cohomology and functors Ext $^{n}$ ) C. R. Acad. Sci. Paris Sr. I Math. 296 (1983), no. 23, 953-958.

[4] A. Connes, Noncommutative differential geometry. Inst. Hautes tudes Sci. Publ. Math. No. 62 (1985), 257-360.

[5] A. Connes, Cyclic cohomology and the transverse fundamental class of a foliation. Geometric methods in operator algebras (Kyoto, 1983), 52-144, Pitman Res. Notes Math. Ser., 123, Longman Sci. Tech., Harlow, 198.

[6] A. Connes, and M. Marcolli, From physics to number theory via noncommutative geometry. Part I: Quantum statistical mechanics of Q-lattices. math.NT/0404128.

[7] A. Connes, and M. Marcolli, From physics to number theory via noncommutative geometry, Part II: Renormalization, the Riemann-Hilbert correspondence, and motivic Galois theory. hep-th/0411114.

[8] A. Connes and H. Moscovici, Local index formula in noncommutative geometry, Geom. Funct. Anal. 5 (1995), no. 2, 174-243. 
[9] A. Connes and H. Moscovici, Hopf algebras, Cyclic Cohomology and the transverse index theorem, Comm. Math. Phys. 198 (1998), no. 1, 199246.

[10] A. Connes and H. Moscovici, Cyclic cohomology and Hopf algebras. Lett. Math. Phys. 48 (1999), no. 1, 97-108.

[11] A. Connes, and H. Moscovici, Cyclic cohomology and Hopf algebra symmetry. Conference Mosh Flato 1999 (Dijon). Lett. Math. Phys. 52 (2000), no. $1,1-28$.

[12] A. Connes and H. Moscovici, Modular Hecke algebras and their Hopf symmetry, to appear in the Moscow Mathematical Journal (volume dedicated to Pierre Cartier).

[13] A. Connes and H. Moscovici, Rankin-Cohen brackets and the Hopf algebra of transverse geometry, to appear in the Moscow Mathematical Journal (volume dedicated to Pierre Cartier).

[14] P. M. Hajac, M. Khalkhali, B. Rangipour, and Y. Sommerhäuser, Stable anti-Yetter-Drinfeld modules. C. R. Math. Acad. Sci. Paris 338 (2004), no. 8, 587-590.

[15] P. M. Hajac, M. Khalkhali, B. Rangipour, and Y. Sommerhäuser, Hopfcyclic homology and cohomology with coefficients. C. R. Math. Acad. Sci. Paris 338 (2004), no. 9, 667-672.

[16] P. Jara, D. Stefan, Cyclic homology of Hopf Galois extensions and Hopf algebras. Preprint math/0307099.

[17] A. Joyal, and R. Street, The geometry of tensor calculus. I. Adv. Math. 88 (1991), no. 1, 55-112.

[18] Ch. kassel, Quantum groups. Graduate Texts in Mathematics, 155. Springer-Verlag, New York, 1995.

[19] A. Kaygun, Bialgebra cyclic homology with coefficients. Preprint math/0408094.

[20] M. Khalkhali, and B. Rangipour, A new cyclic module for Hopf algebras. K-Theory 27 (2) (2002), 111-131. 
[21] M. Khalkhali, and B. Rangipour, Invariant cyclic homology. K-Theory 28 (2) (2003), 183-205.

[22] M. Khalkhali, and B. Rangipour, A note on cyclic duality and Hopf algebras. Comm. in Algebra 33 (2005) 763-773.

[23] M. Khalkhali, and B. Rangipour, Cup products in Hopf-cyclic cohomology. C.R. Acad. Sci. Paris Ser. I 340 (2005), 9-14.

[24] M. Marcolli, Lectures on noncommutative arithmetic geometry. math.QA/0409520

[25] S. Mac Lane, categories for the working mathematician. Second edition. Graduate Texts in Mathematics, 5. Springer-Verlag, New York, 1998.

[26] S. Majid, Foundations of quantum group theory. Cambridge University Press, Cambridge, 1995.

[27] D. Radford, and J. Towber, Yetter-Drinfeld categories associated to an arbitrary bialgebra. J. Pure Appl. Algebra 87 (1993), 259-279.

[28] M. Staic, personal communication.

[29] M. Sweedler, Hopf algebras. Mathematics Lecture Note Series W. A. Benjamin, Inc., New York 1969.

[30] R. Taillefer, Cyclic Homology of Hopf Algebras. K-Theory 24 (2001), no. $1,69-85$.

[31] C. Voigt, personal communication.

[32] D. Yetter, Quantum groups and representations of monoidal categories. Math. Proc. Cambridge Philos. Soc. 108 (1990), no. 2, 261-290. 